\title{
New ionic liquids for modification of chitin particles
}

\author{
Malgorzata M. Jaworska ${ }^{1}$ Andrzej Górak ${ }^{2,3}$
}

Received: 13 April 2017/ Accepted: 18 June 2017/Published online: 3 March 2018

(C) The Author(s) 2018. This article is an open access publication

\begin{abstract}
Chitin particles are chemically and mechanically stable due to the strong inter- and intra-sheet network of hydrogen bonds and the large number of crystalline regions. Thus, it is difficult to make any modification of the chitin particles themselves. Therefore, new solvents which can modify the structure of chitin particles are being sought. This work presents the influence of unreported ionic liquids (ILs) containing the TCB anion on chitin: three of them contained the imidazolium ring, ([Im], [Dmim], [EOHmim]) and another three contained the pyridinum ring ([Pyr], [EOHpyr], [POHpyr]). Chitin did not dissolve in any of the tested ILs but the ILs were able to modify the structure (porosity and corrugation) of chitin particles. It has also been shown that [EOHpyr][TCB] caused the degradation of chitin particles. The degradation process strongly depends on temperature and the time of interaction; choosing proper conditions, particles with a narrow particle size distribution could be obtained.
\end{abstract}

Keywords Chitin · Imidazolium · Pyridinum · TCB · Ionic liquid · Particle morphology

Malgorzata M. Jaworska

malgorzata.jaworska@pw.edu.pl

1 Faculty of Chemical and Process Engineering, Warsaw University of Technology, ul. Warynskiego 1, 00-645 Warsaw, Poland

2 Faculty of Biochemical and Chemical Engineering, Technical University of Dortmund, Dortmund, Germany

3 Faculty of Process and Environmental Engineering, Lodz University of Technology, Lodz, Poland 


\section{Introduction}

Ionic liquids (ILs) capable of dissolving or modifying chitin or chitosan particles have recently been intensively investigated. Their ability to dissolve chitin is extremely interesting as it gives the opportunity to apply solvents that are less toxic and less corrosive than those presently used, like $\mathrm{LiCl} /$ dimethylacetamide, concentrated $\left(16 \%\right.$ ) cold (around $0{ }^{\circ} \mathrm{C}$ ) $\mathrm{NaOH}$ solution or $\mathrm{CaCl}_{2} \cdot \mathrm{H}_{2} \mathrm{O}$ saturated with methanol [1, 2]. ILs can be used for the modification of chitin particles to increase the porosity or to increase the external surface area by their corrugation [3]. The modification should be focused to create particles with a large, expanded surface as there will be a larger number of binding centers in comparison to untreated chitin. Such particles (with a large external surface area) can then be used to prepare new carriers for enzymes or a new matrix for chromatography. It is difficult to prepare such particles using typical solvents for the chitin.

Searching for a good solvent for chitin or an IL able to modify the structure of chitin particles, we have tested several typically used ILs (with imidazolium cation [3]) as well as some not mentioned in literature previously (as with pyridinum cation or with anions different from halide or organic acid). In our investigations we have observed an interesting interaction between chitin and ILs containing the TCB (tetracyanoborate) anion and imidazolium or pyridinum cationa. According to our best knowledge, these ILs have not to date been reported in the literature for the modification of chitin particles.

The aim of the present paper is to show the influence of selected ILs containing the TCB anion on chitin particles. Six ILs, all containing the TCB anion and with two types of ring in a cation have been tested: three of them contained the imidazolium ring (imidazolium, [Im]; 1,2-dimethylimidazolium, [Dmim]; 1-(2hydrohyethyl)-3-methylimidazolium, [EOHmim]), and three of them contained the pyridinum ring (pyridinum, [Pyr]; 1-(2-hydroxyethyl)pyridinum, [EOHpyr]; 1-(2hydroxypropyl)pyridinum [POHpyr]). These ILs let us compare the influence of the kind of ring ([Im] vs. [Pyr], and [EOHmim] vs. [EOHpyr]) as well as kind of substituent of the rings ([Im] vs. [Dmim] vs. [EOHmim] and [Pyr] vs. [EOHpyr] vs. [POHpyr]).

As the main goal of our investigation is to find the ILs that would be able to change the structure of chitin particles, we have focused our investigations on the solubility of chitin in ILs, changes in the structure of chitin particles observed under electron microscope (SEM) and changes in particle size distribution (Laser Diffraction Particle Size Analyzer). ILs are still expensive compounds so we also checked the possibility of the reuse of ILs several times for chitin modification; changes in the ILs were evaluated on the basis of FTIR spectra. Additionally, the influence of process conditions (initial size of the chitin particles, temperature and time of chitin incubation) on changes in particle size distribution have been presented for selected ILs. 


\section{Materials and methods}

\section{Chemicals}

a-Chitin from shrimp, with an acetylation degree of $96 \%$, was purchased from BioLog Heppe (Germany). Particles were ground and a fraction of 25-50 $\mu \mathrm{m}$ was used unless otherwise mentioned.

All ILs: imidazolium tetracyanoborate ([Im][TCB]), 1,2-dimethylimidazolium tetracyanoborate ([Dmim][TCB]), 1-(2-hydroxyethyl)-3-methylimidazolium tetracyanoborate ([EOHmim][TCB]), pyridinum tetracyanoborate ([Pyr][TCB]), 1-(2hydroxyethyl)pyridinum tetracyanoborate ([EOHpyr][TCB]), and 1-(2-hydroxypropyl)pyridinum tetracyanoborate ([POHpyr][TCB]) were kindly donated by Merck.

All chemicals, including ethanol and water, were of analytical grade.

\section{Experimental}

\section{Solubility of chitin in $I L$}

Ionic liquid (approx. 1.0-1.5 g) was weighed into a test tube, heated $\left(105^{\circ} \mathrm{C}\right)$ and mixed $(200 \mathrm{rpm})$ and small portion (approx. $0.05 \%$ of the mass of ionic liquid) of chitin was added. Samples were left for 24-48 h.

Dissolution was assessed visually (a transparent solution was expected) and under light microscope (Fig. 1).

\section{Influence of ILs on particles of chitin}

The ionic liquid (approx. $2.0 \mathrm{~g}$ ) was weighed into a test tube and then chitin $(5 \%$ w/w) was added. Samples were incubated in an oil bath at $105^{\circ} \mathrm{C}$ mixing $(200 \mathrm{rpm})$
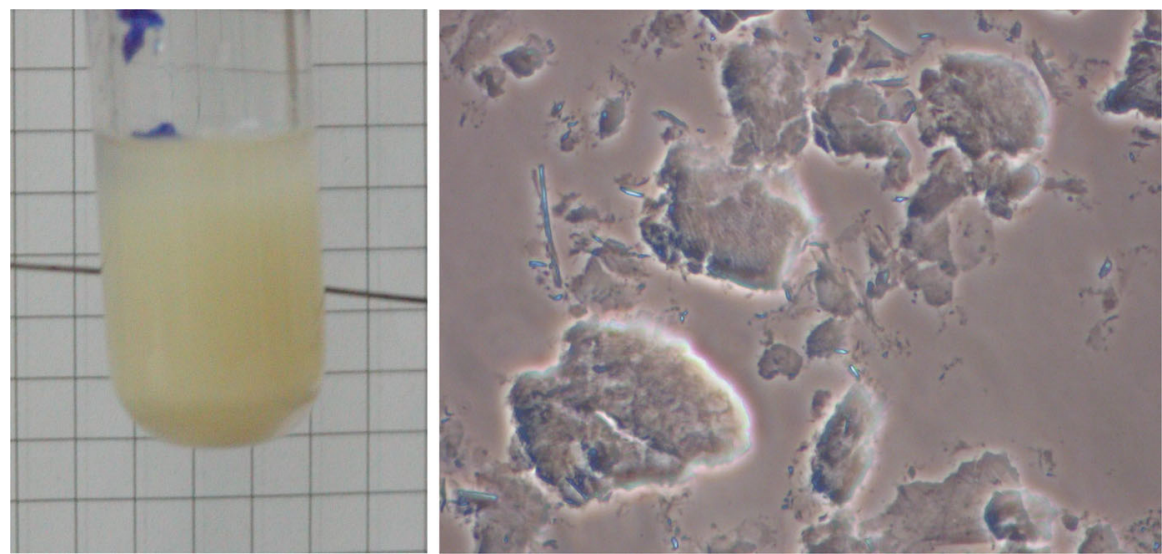

Fig. 1 Suspension of chitin in ionic liquid has been assessed visually and under light microscope 
for $48 \mathrm{~h}$ (unless different conditions are mentioned). Then, the chitin was recovered by adding the chitin suspension in the $\mathrm{IL}$ dropwise into hot $\left(95^{\circ} \mathrm{C}\right)$ water, whereupon chitin particles precipitated from the solution. These particles were left in the solution until full sedimentation, then part of the liquid was removed with an automatic pipette (up to $50 \%$ of the total volume), and a fresh ethanol/water sample (the same volume as was taken out) was added and mixed in a vortex-type mixer. Chitin particles were washed 5-7 times with ethanol and then 3-5 times with water (the suspension was left until full sedimentation of the particles after each wash, before a new portion of ethanol/water was added). Finally, chitin particles were lyophilized.

\section{Reuse of ionic liquid}

All solutions recovered from washing out the chitin (all portions of ethanol and water) were collected in test tubes and dried by lyophilisation. The ionic liquid was then reused in the same way as described above (section 2.2.2.). Each sample of the ionic liquid was used 4 times.

\section{Analytical methods}

\section{Particle size distribution}

The particle size distribution $\left(d_{10 \%}-d_{90 \%}\right)$ and mean size of the particles $\left(d_{\text {mean }}\right)$ were evaluated using a Laser Diffraction Particle Size Analyzer (LS 13,320 Universal Liquid Module; Coulter-Beckman, USA), a mean value of 3 samples being taken. The standard deviation (SD) of $d_{\text {mean }}$ was lower than $10 \%$.

\section{Electron microscopy}

A Nikon ELWD 03/OD75 Electron Microscope (Japan) was used for the SEM observations and photographs of the samples that had been lyophilized and sprayed with gold were taken. Typical observations of at least 3-5 preparations are presented.

\section{FTIR spectra}

FTIR spectra were obtained using a Thermo Scientific Nicolet 6700 FTIR Spectrophotometer (USA). The ILs were measured directly. All scans were carried out from 4000 to $400 \mathrm{~cm}^{-1}$ with a resolution of $6 \mathrm{~cm}^{-1}$ as the mean of 32 scans.

FTIR spectra of chitin (after each series) were measured as 13-mm discs with $\mathrm{KBr}(1 \mathrm{mg}$ :200 mg). All scans were taken using the same apparatus in the range of wavelengths $4000-400 \mathrm{~cm}^{-1}$ with a resolution of $6 \mathrm{~cm}^{-1}$. The mean of 32 scans was presented as the result. 


\section{Results}

Six ILs were tested as solvents for chitin. We assume that most of them were able to modify the particle size or structure of chitin particles. In the first part of our work, the solubility of chitin in the tested ILs was investigated. Next, the influence of the ILs on the structure of chitin particles as well as the possibility of reuse of the ILs was investigated. Each sample of each IL was used 4 times (denoted as $1 s, 2 s, 3 s$, $4 s$ ), where each series consisted of a mixture of chitin in the IL, recovered with water (added dropwise to hot water), and the washing out of the chitin with ethanol, then with water, followed by the evaporation of the ethanol/water from the IL.

\section{Solubility of chitin in ionic liquids}

The solubility of chitin in the tested ILs was assessed on the basis of the transparency of the sample (visual observations) and on the basis of observations under a light microscope. None of the tested ILs was able to create a transparent solution for a chitin concentration as low as $0.5 \% \mathrm{w} / \mathrm{w}$. In all cases, a suspension of chitin in the IL was observed. This means that the chitin solubility was less than $0.5 \%$, so we can assume that chitin is insoluble in the tested ILs containing the TCB anion.

For each chitin sample, after recovery, FTIR spectra were examined to check for the presence of ILs. The washing out of the ILs from the chitin samples was confirmed by the comparison of spectra of recovered and untreated chitin; no changes in chitin spectra were detected.

\section{SEM observations}

Chitin was regenerated from IL solutions by dropwise adding to hot water. Typical changes in the structure of chitin particles are presented in Fig. 2.

Depending on the IL used, different changes in the regenerated chitin particles were observed. For the chitin particles treated first with [Im][TCB] $(1 s)$, an increase in porosity was observed, while for every subsequent series, the surface was more cracked and, for the final series $(4 s)$, the surface looked like shells. Similar behaviour was observed for chitin treated with [Dmim][TCB] and [EOHmim][TCB]. Particles (after the first series $1 s$ ) were more porous, while with each subsequent series the pores changed into "cracks". It seems that, with every succeeding series, the IL destroyed more and more hydrogen bonding in the chitin particles.

The [Pyr][TCB] IL was also able to modify chitin particles: no changes were observed for the first series while with every subsequent series increases in corrugation and porosity were noticed. Substituting the ring with the POH group first caused an increase in porosity $(1 s)$, the next $(2 s)$ an increase in corrugation of the surface, but the corrugation was smaller with each subsequent series and finally, for $(4 s)$, no significant changes were observed. 


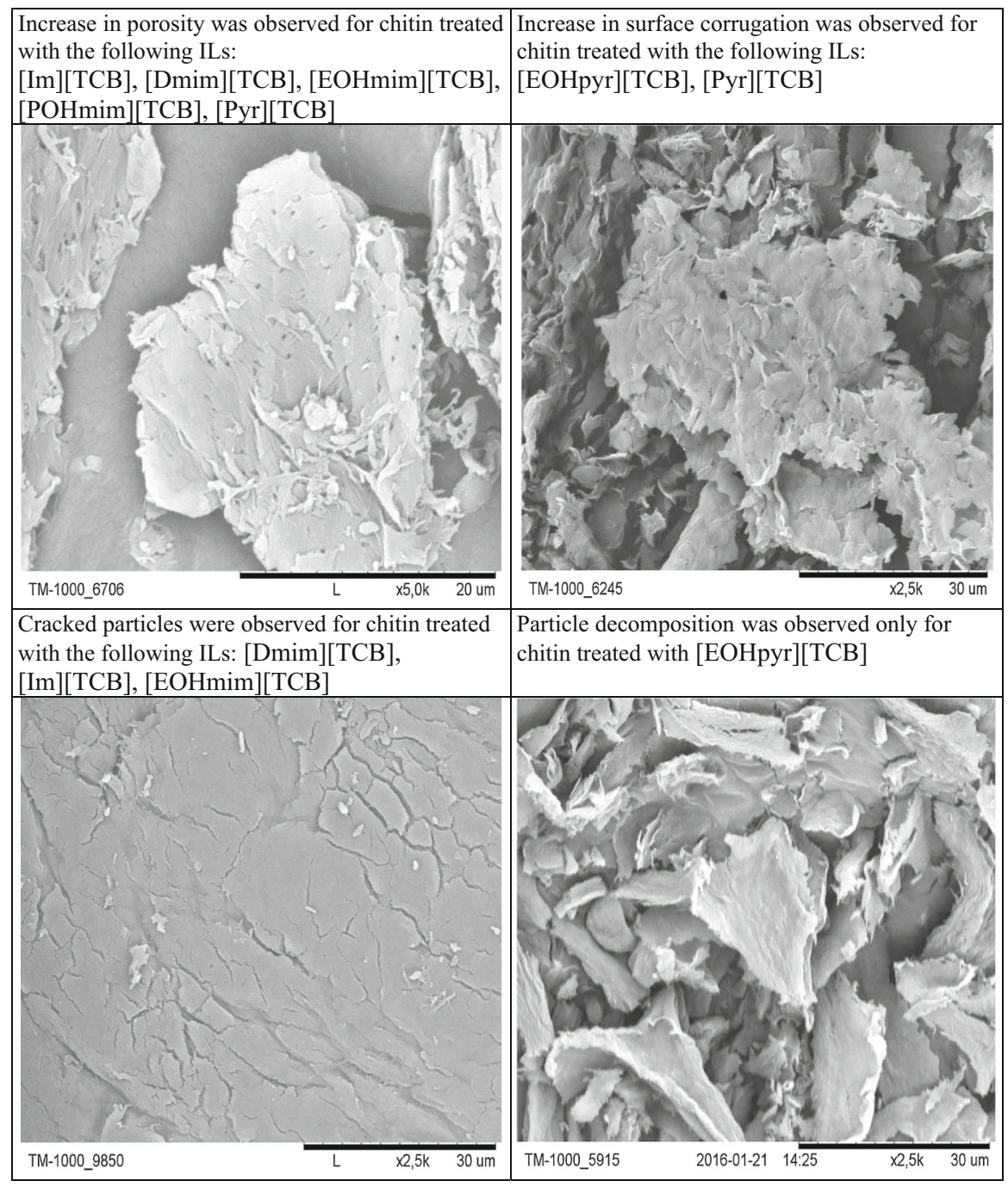

Fig. 2 Typical changes in the structure of recovered chitin particles

Different changes were noticed for [EOHpyr][TCB] IL. This IL was able not only to change the structure of the particle surface but also to destroy the particles, as a significant decrease in particle size was observed under SEM. Chitin was insoluble in this IL and also no gelation was observed. This IL acting on solid particles caused their destruction to smaller ones. This observation was confirmed by particle size distribution measurement. 


\section{Particle size distribution}

ILs can change not only the structure of chitin particles but can also influence the particle size. The particle size distribution of chitin particles regenerated by dropwise addition to hot water was compared. Mean value $\left(d_{\text {mean }}\right)$ and particle size distribution $\left(d_{10 \%}-d_{90 \%}\right)$ are shown in Table 1.

The presented data show that only for chitin treated with [EOHpyr][TCB] was a change (decrease) in chitin particle size observed and particles with a much smaller size were obtained after recovery. In order to prove that the effect is not caused by the method of recovery, the prepared chitin suspension in IL was also mixed with hot water $\left(95^{\circ} \mathrm{C}\right.$ ) in a vortex-type mixer (see Table 2 ). Chitin particles with a smaller size than the original ones were also obtained. As the chitin did not dissolve in [EOHpyr][TCB] IL, the observed decrease in particle size was due to the interaction of solid particles with the IL. This effect was caused by interaction rather with the cation [EOHpyr] as all ILs used in the experiments contained the same anion [TCB]. Degradation of chitin particles was also observed for the IL containing [EOHmim] cations where the imidazolium ring was substituted by the hydroxyethyl group, but this effect was less significant. Comparing cations with the imidazolium ring shows that only the hydroxyethyl substituent caused degradation of particles. The [Im] and [Dmim] cations did not influence the particle size. The same observations could be carried out for cations with the pyridinum ring. The [Pyr] cation did not influence the particle size, while the IL with the [POHpyr] cation gave particles that were slightly smaller than untreated chitin. Comparing the influence of the substituent of the pyridinum ring ([EOHpyr] and [POHpyr]), the decrease in particle size was observed only for the [EOHpyr] cation. As the degradation effect was noticed only for the [EOHpyr] and [EOHmim] cations, the influence of the hydroxyethyl substituent on the degradation process can be assumed. This effect

Table 1 Mean size of chitin particles $\left(d_{\text {mean }}\right)$ and particle size distribution $\left(d_{10 \%}-d_{90 \%}\right)$

\begin{tabular}{|c|c|c|c|c|}
\hline & $1 s$ & $2 s$ & $3 s$ & $4 s$ \\
\hline & $\begin{array}{l}d_{10 \%}-d_{90 \%} \\
\left(d_{\text {mean }}\right)[\mu \mathrm{m}]\end{array}$ & $\begin{array}{l}d_{10 \%}-d_{90 \%} \\
\left(d_{\text {mean }}\right)[\mu \mathrm{m}]\end{array}$ & $\begin{array}{l}d_{10 \%}-d_{90 \%} \\
\left(d_{\text {mean }}\right)[\mu \mathrm{m}]\end{array}$ & $\begin{array}{l}d_{10}-d_{90 \%} \\
\left(d_{\text {mean }}\right)[\mu \mathrm{m}]\end{array}$ \\
\hline Untreated chitin & \multicolumn{4}{|l|}{$34-126(74)$} \\
\hline$[\mathrm{Im}][\mathrm{TCB}]$ & $20-144(78)$ & $22-145(81)$ & 21-147 (80) & $20-138(76)$ \\
\hline$[\mathrm{Dmim}][\mathrm{TCB}]$ & 23-138 (77) & 23-138 (77) & $21-142(78)$ & $21-146(80)$ \\
\hline$[$ EOHmim $][\mathrm{TCB}]$ & $15-106(54)$ & $15-92(56)$ & $16-115(61)$ & $15-95(51)$ \\
\hline [Pyr][TCB] & $24-135$ (75) & $22-144$ (79) & $18-122(66)$ & $20-129(73)$ \\
\hline \multirow[t]{2}{*}{ [EOHpyr][TCB] } & $3-46(18)$ & $3-36(18)$ & $9-54(29)$ & $11-68(38)$ \\
\hline & $5-106(48)$ & $7-89$ (44) & $9-74(36)$ & $12-95$ (46) \\
\hline [POHpyr][TCB] & $18-128(68)$ & $22-132(75)$ & $19-132(71)$ & $19-121(67)$ \\
\hline
\end{tabular}

$\mathrm{SD}<10 \%$ for $d_{\text {mean }}, d_{10 \%}, d_{90 \%}$

${ }^{\text {a }} d$ dropwise to hot water, $v$ mixed with hot water in a vortex-type mixer 
could be caused not only by the presence of the hydroxyl group (degradation observed for [EOHpyr] and [POHpyr]) but mainly by the ethyl group, as the ILs with ethyl substituent in the imidazolium ring [Emim] have been reported as able to dissolve chitin or to modify its particles [4-8].

\section{FTIR spectra of ILs}

FTIR spectra of original ILs and ILs after recovery in the last series $(4 s)$ were compared. We have chosen the ILs recovered after the last series of experiments as, for these ILs, any possible changes caused by decomposition/changes in the structure as well as impurity accumulation should be easily observed. The comparison of spectra is presented in Fig. 3.

Comparing the FTIR spectra of the original ILs and those after recovery from the $4 s$ process, no significant changes in spectra were observed. The only changes noticed were in intensity of the peaks, but no additional or disappeared peaks were noticed. Even for the [EOHpyr][TCB]-4s spectrum, no additional peaks characterized for chitooligomers have been noticed, which suggests that the particles have not been degraded to oligomers.

\section{Parameters influencing modification of chitin particles with [EOHpyr][TCB]}

The [EOHpyr][TCB] IL was able to destroy hydrogen bonds between chitin chains and caused degradation of chitin particles. The degree of degradation depends on process parameters such as: initial particle size, temperature of treatment and time of incubation. The influence of these parameters has been tested and is presented below. Although the presented data are rather qualitative, they can be the basis for further quantitative investigations.

\section{Influence of initial particle size}

The influence of initial particle size on the degradation of chitin particles has been investigated with two chitin fractions differing in the $d_{\text {mean }}$. Chitin particles with initial mean sizes of 368 and $74 \mu \mathrm{m}$ were used. The particles were treated with [EOHpyr][TCB] IL for $48 \mathrm{~h}$ at $102{ }^{\circ} \mathrm{C}$ and were recovered by adding dropwise to hot water (1s) (Fig. 4).

Chitin particles have been degraded regardless of the initial particle size of the chitin. Particles with the initial size $d_{\text {mean }}=74 \mu \mathrm{m}$ have been degraded to particles of $d_{\text {mean }}=18 \mu \mathrm{m}$, while the particles with $d_{\text {mean }}=368 \mu \mathrm{m}$ have been degraded to $d_{\text {mean }}=63 \mu \mathrm{m}$. The change in particle size was $76 \%$ for smaller particles and $83 \%$ for larger particles, which can suggest that larger particles are more easily degraded than smaller ones. Larger particles could contain probably higher numbers of discontinuity points or larger numbers of amorphous regions in the particles so that their hydrogen bond could be more easily broken. This effect was caused by the IL 

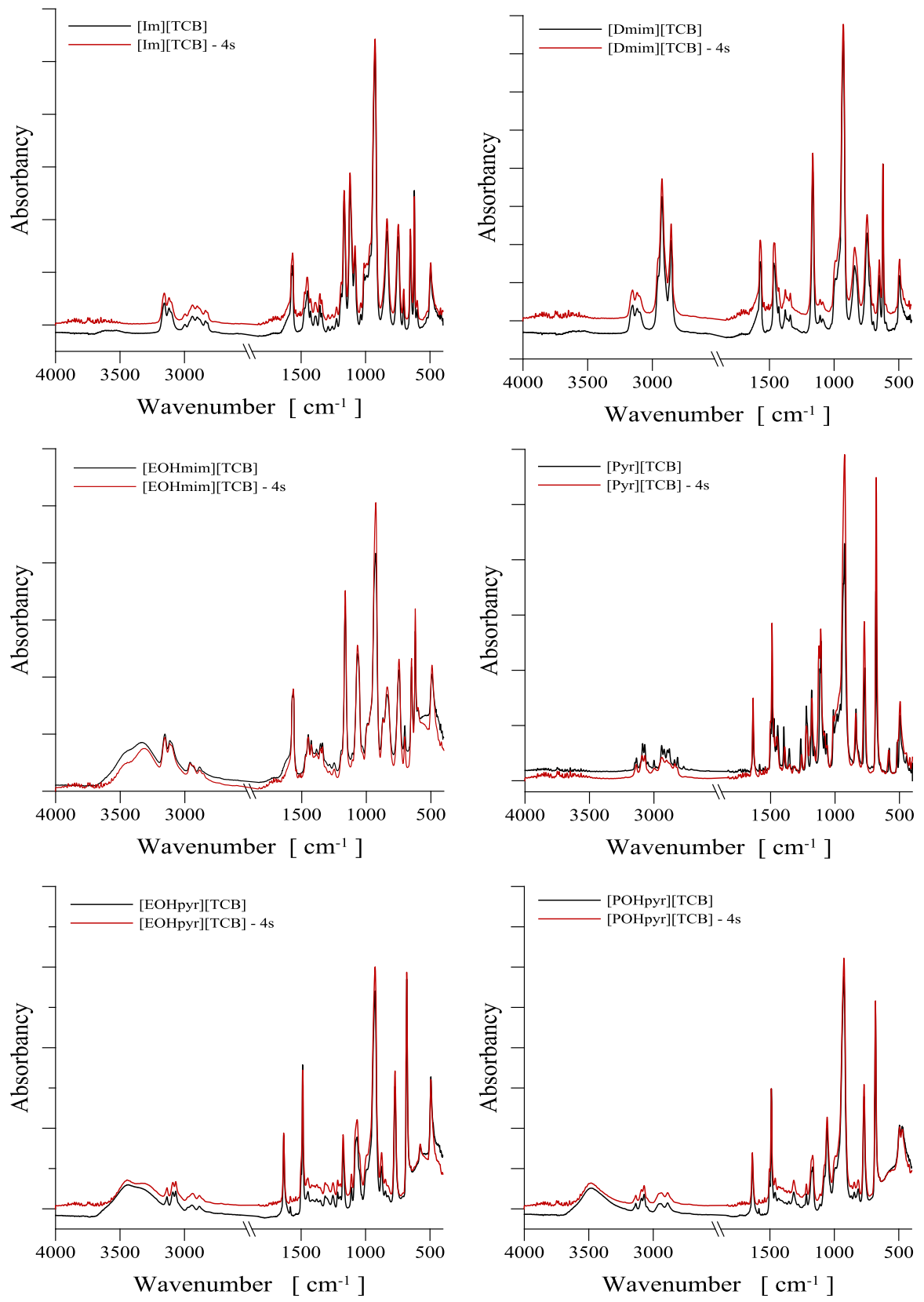

Fig. 3 FTIR spectra of tested ILs; black line new IL, red line ILs recovered after $4 \mathrm{~s}$

used, as the chitin samples with the same particle size distribution treated with water in the same conditions did not show any changes in particle size distribution or mean particle size. 

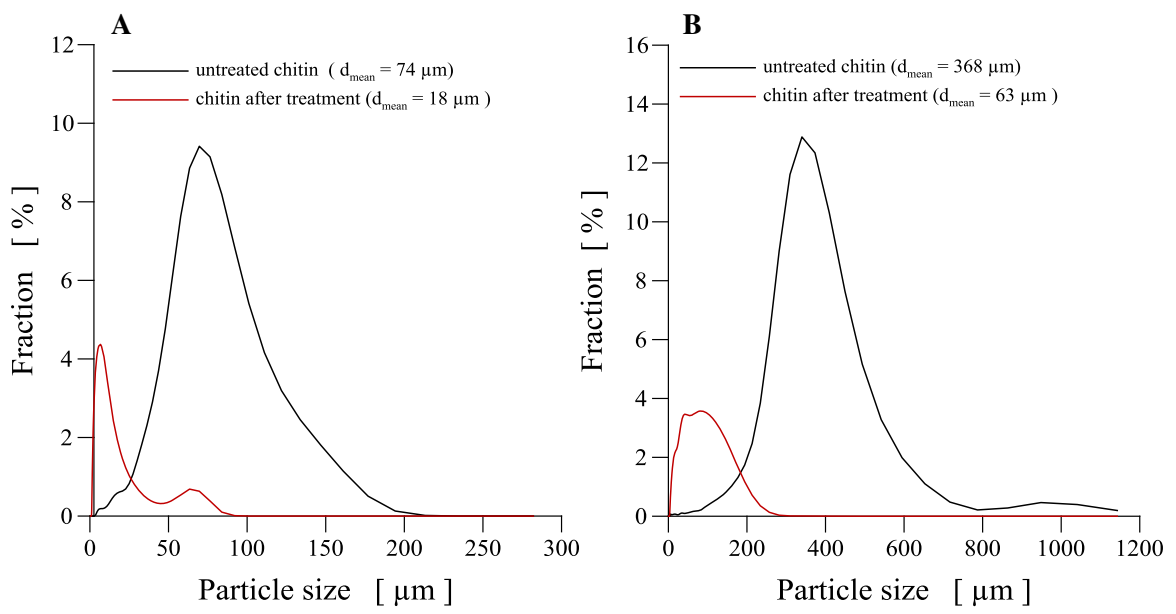

Fig. 4 Comparison of particle size distribution of untreated chitin and chitin after recovery from [EOHpyr][TCB] (1s); initial particle size $d_{\text {mean }} 74 \mu \mathrm{m}(\mathbf{a})$ and $368 \mu \mathrm{m}(\mathbf{b})$

\section{Influence of temperature}

Usually, temperatures above $100{ }^{\circ} \mathrm{C}$ have been applied for the investigation of chitin interaction with ILs. Several ILs are highly hydroscopic and such temperatures are useful as traces of water will evaporate. Additionally, higher temperatures will increase the rate of dissolution of chitin. The increase of temperature cannot be too high as chitin can degrade or the process cannot be economically worthwhileas heating would increase the cost of the process. In our investigations, the temperatures of 105 and $124{ }^{\circ} \mathrm{C}$ have been tested (1s) (Fig. 5; Table 2).

In the experiments, the fraction of chitin with a large particle size was used. The temperature of incubation influenced the size of the chitin particles obtained after the treatment. Contrary to our expectations, a higher temperature was not favorable as three peaks corresponding to three different particle sizes $(150,350$ and $480 \mu \mathrm{m})$ were noticed in the spectrum. For a lower temperature $\left(102{ }^{\circ} \mathrm{C}\right)$, the narrow particle size distribution was noticed with a large maximum at $130 \mu \mathrm{m}$ and a much smaller at $213 \mu \mathrm{m}$. For higher temperatures, a larger content of large particles was observed, which could have been caused not by the creation of agglomerates, but by the degrading process of the largest particles. The data suggest that a lower temperature is more favorable when particles with a narrow particle range of distribution are needed. This observation is significant as usually a wide range of particles is obtained by the grinding and sieving process, as can be seen for untreated chitin. 


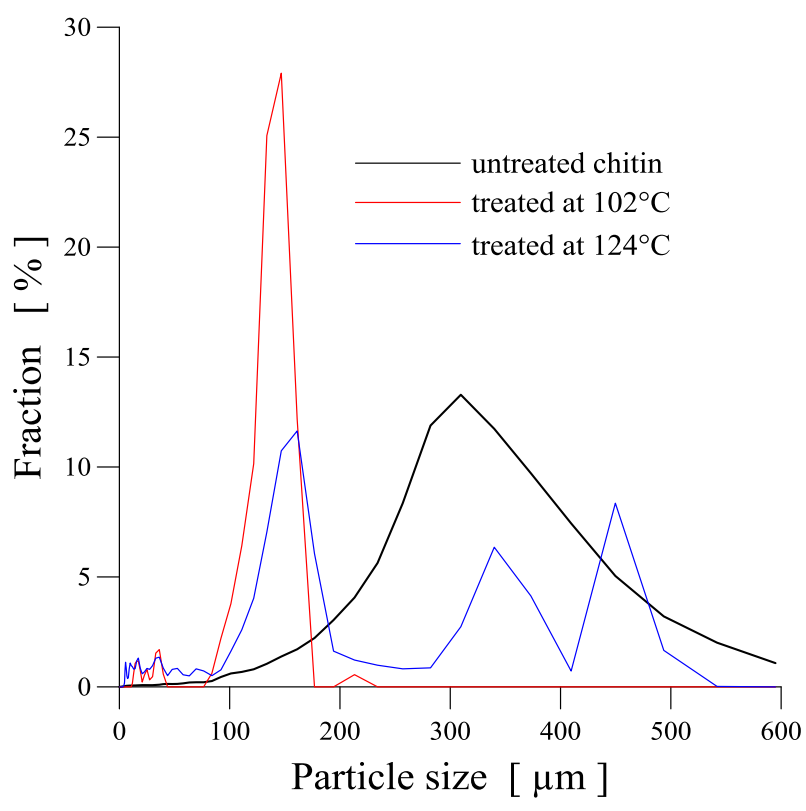

Fig. 5 Particle size distribution after treatment with [EOHpyr][TCB] at different temperatures $(1 s)$

Table 2 The influence of temperature on modification of chitin particles with [EOHpyr][TCB], $(1 s)$

\begin{tabular}{|c|c|c|}
\hline Untreated chitin & $\begin{array}{r}\mathrm{d}_{10 \%}-\mathrm{d}_{90^{\circ}} \\
\mathrm{d}_{\text {mean }} \\
\end{array}$ & $\begin{array}{c}210-528 \mu \mathrm{m} \\
368 \mu \mathrm{m}\end{array}$ \\
\hline \multicolumn{3}{|c|}{+ + [EOHpyr][TCB] } \\
\hline & $102^{\circ} \mathrm{C}$ & $124^{\circ} \mathrm{C}$ \\
\hline $\mathrm{d}_{10 \%}-\mathrm{d}_{90 \%,},[\mu \mathrm{m}]$ & $59-164$ & $16-420$ \\
\hline $\mathrm{d}_{\text {mean }},[\mu \mathrm{m}]$ & 130 & ----- \\
\hline
\end{tabular}

\section{Influence of incubation time}

The influence of incubation time was investigated using particles with a mean size of $d_{\text {mean }}=325 \mu \mathrm{m}$. The process was carried out for 1,2 or 7 days at $102{ }^{\circ} \mathrm{C}$. Comparisons of particle size distribution $(1 s)$ are presented in Fig. 6 and Table 3.

The presented data showed that incubation time plays important role in the degradation of chitin particles. Alter 1 day of treatment (approx. $24 \mathrm{~h}$ ), the effect was similar to that observed for incubation at $124{ }^{\circ} \mathrm{C}$, as the two maxima (194 and $340 \mu \mathrm{m}$ ) were recorded. For a longer time, 2 days (approx. $48 \mathrm{~h}$ ), one large peak at $130 \mu \mathrm{m}$ accompanied by another much smaller at $220 \mu \mathrm{m}$ have been observed. Finally, for 7-days of incubation, significant degradation of chitin particles was noticed and the distribution pattern became flat with large numbers of particles in the range from 6 to $200 \mu \mathrm{m}$. Even for such a long time of incubation, no transparency of the sample was observed and observations under a light microscope 


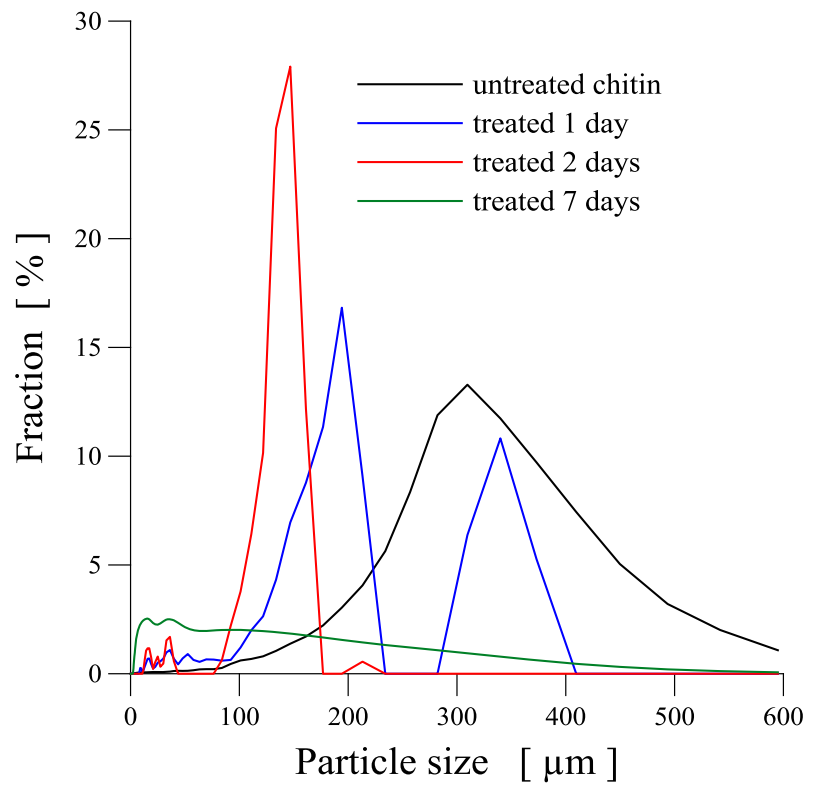

Fig. 6 Particle size distribution of chitin particles after treatment (1s)

Table 3 Influence of incubation time on changes in particle size distribution of chitin (1s)

\begin{tabular}{|c|c|c|c|}
\hline Untreated chitin & \multicolumn{3}{|c|}{$\begin{array}{cc}\mathrm{d}_{10 \%}-\mathrm{d}_{90 \%} & 176-468 \mu \mathrm{m} \\
\mathrm{d}_{\text {mean }} & 325 \mu \mathrm{m}\end{array}$} \\
\hline \multicolumn{4}{|c|}{$+[$ EOHруr] $]$ ТСВ $]$} \\
\hline & 1 day & 2 days & 7 days \\
\hline $\begin{array}{l}\mathrm{d}_{10 \%}-\mathrm{d}_{90 \%} \\
{[\mu \mathrm{m}]}\end{array}$ & $73-285$ & $59-164$ & $6-196$ \\
\hline $\begin{array}{l}\mathrm{d}_{\text {mean }} \\
{[\mu \mathrm{m}]}\end{array}$ & 194 and 340 & 130 & 71 \\
\hline
\end{tabular}

were similar to those presented in Fig. 1. The viscosity of the samples (assessed visually) was no different than that for the sample treated for $24 \mathrm{~h}$. On the basis of these observations, we can assume that chitin was rather degradated to small particles than partially dissolved in [EOHpyr][TCB]. 


\section{Conclusions}

In this paper, the influence of six ILs containing the TCB anion on the solubility, the change in the structure of chitin particles and their particle size distribution is presented. The possibility of reuse of ILs for modification of chitin particles has also been tested.

ILs with the [TCB] anion were not able to dissolve or gel chitin particles, but they modified their structure, as particles with cracks, increased porosity and increased corrugation have been observed under SEM. These modifications were independent of whether freshly synthesized or reused IL was applied. The change in particle structure, especially increase in surface corrugation and increase in porosity, will increase the external surface area. Therefore, such modified particles will be characterized by an increased number of binding sites. This will be advantageous from the point of view of its use as a carrier for enzymes or chromatographic applications.

Additionally, no differences have been observed in the FTIR spectra of fresh and reused ILs which means that ILs can be applied several times without any harm. Since no additional peaks in the FTIR spectra were observed, the degradation of chitin particles caused by [EOHpyr][TCB] or [EOHmim][TCB] ILs did not generate any chitin oligomers.

Among tested ILs, the strong effect of the degradation of chitin particles was observed for [EOHpyr][TCB]. That degradation was probably related to the weakening/destruction of the hydrogen bonds among the chitin chains in the amorphous regions rather than hydrolysis of a single chitin chain at the surface of its particles. Comparing the data for the tested ILs, we can assume that the degradation of the chitin particles was related to the presence of ethyl and hydroxyl groups in the substitution of the pyridinum or imidazolium ring. The process depended on temperature and incubation time. This observation is valuable as, taking the chitin particles with certain initial particle size distributions and processing them at a proper temperature and during a proper time, particles with predicted size and narrow particle size distribution could be obtained, which is difficult to achieve when the particles are ground in a mill and sieved. Narrow particle size distribution is an important parameter for the production of the carriers for enzymes or particles for chromatographic applications. Taking into account that [EOHpyr][TCB] IL also caused an increase in the surface corrugation of modified chitin particles, and thus also increases in the external surface area and the number of binding sites, it can be considered as a good agent for chitin modification in the mentioned applications. Thus, the presented data have confirmed that it is possible to modify the structure or size of chitin particles with the ILs used here.

Acknowledgements This work has been supported by the budget sources for The National Centre for Science (Poland), Grant No. DEC-2013/09/B/ST8/00144. The authors would like to thank Merck KGaA for their kind donation of all the ionic liquids.

Open Access This article is distributed under the terms of the Creative Commons Attribution 4.0 International License (http://creativecommons.org/licenses/by/4.0/), which permits unrestricted use, distribution, and reproduction in any medium, provided you give appropriate credit to the original 
author(s) and the source, provide a link to the Creative Commons license, and indicate if changes were made.

\section{References}

1. G.A.F. Roberts, Chitin Chemistry (Palgrave Macmillan, Basingstoke, 1992)

2. M. Mucha, Chitozan-wszechstronny polimer ze źródeł odnawialnych, WNT (2010)

3. M.M. Jaworska, A. Gorak, Mat. Lett. 164, 341 (2016)

4. M.M. Jaworska, T. Kozlecki, A. Gorak, J. Polym. Eng. 32, 67 (2012)

5. M.G. Hanley, J.M. Green, P.A. Fylstra, D.M. Fox, W.A. Henderson, H.C. de Long, P.C. Trulove, in 210th Meeting of Electrochem. Soc., Cancun (Mexico), Conference Materials, Abstract 2018 (2016)

6. Y. Qin, X. Lu, N. Sun, R.D. Rogers, Green Chem. 12, 968 (2010)

7. J. Shamshina, O. Zavgorodnya, J.R. Bonner, G. Gurau, T. Di Nardo, R.D. Rogers, Chemsuschem 10, 106 (2017)

8. J.L. Shamshina, P.S. Barber, G. Gurau, C.S. Griggs, R.D. Rogers, ACS Sustain. Chem. Eng. 4, 6072 (2016) 